\title{
USO DE MICROHÁBITATS, ACTIVIDAD DIARIA Y DIETA DE DENDROBATES TRUNCATUS (COPE, 1861) (ANURA: DENDROBATIDAE) EN BOSQUE SECO TROPICAL DEL NORTE DE COLOMBIA
}

\author{
MICROHABITAT USE, DAILY ACTIVITY AND DIET OF DENDROBATES \\ TRUNCATUS (COPE, 1861) (ANURA: DENDROBATIDAE) IN TROPICAL \\ DRY FOREST IN NORTHERN COLOMBIA
}

\author{
Carolina POSSO-PELÁEZ, ${ }^{1}$ Argelina BLANCO-TORRES ${ }^{1,2, *}$ y Luis C. GUTIÉRREZ-MORENO ${ }^{1}$ \\ ${ }^{1}$ Grupo de investigación en Biodiversidad del Caribe Colombiano. Facultad de Ciencias Básicas. Universidad del \\ Atlántico. Km 7 Antigua vía a Puerto Colombia, Barranquilla, Colombia. <rotifero15@gmail.com>, <caroposso@ \\ gmail.com>. \\ ${ }^{2}$ Grupo de Investigación: Investigaciones Territoriales para el Uso y Conservación de la Biodiversidad. Carrera 4 \\ No 58- 59, Bogotá, Colombia. <argelinab@gmail.com>. \\ * Autor para correspondencia: <argelinab@gmail.com>. \\ Recibido: 09/03/2017; aceptado: 20/06/2017. \\ Editor responsable: Gustavo Aguirre.
}

Posso-Peláez, C., Blanco-Torres, A. y Gutiérrez-Moreno, L. C. (2017). Uso de microhábitats, actividad diaria y dieta de Dendrobates truncatus (Cope, 1861) (Anura: Dendrobatidae) en bosque seco tropical del norte de Colombia. Acta Zoológica Mexicana ( $n$. s.), 33(3), 490-502.

RESUMEN. Se estudió el uso de microhábitats, actividad diaria y dieta de tres poblaciones de Dendrobates truncatus en el bosque seco tropical de tierras bajas del Caribe colombiano, mediante la utilización del método de encuentro visual y captura manual. El estudio se realizó entre los meses de marzo y noviembre, durante los tres períodos climáticos del año: seco, lluvias menores y lluvias mayores. Se identificaron y muestrearon siete microhábitats usados por esta especie, la actividad se midió a través de la relación número de individuos/hora en cuatro horarios diarios. Se examinaron los contenidos estomacales de los anuros y se aplicó el método de análisis porcentual. D. truncatus presenta mayor actividad en la mañana (8:00 - 10:00) y final de la tarde (16:00 - 18:00) y usa siete tipos de microhábitats, preferentemente los de cobertura boscosa y con altos valores de humedad. Presenta preferencia marcada por el consumo de hormigas, especialmente la familia Myrmicinae y el género Pheidole. Estos resultados sugieren que la conservación de relictos de bosque seco tropical y el desarrollo de estrategias de rehabilitación del mismo se constituyen en elementos fundamentales para la supervivencia de esta especie.

Palabras clave: Espacio, tiempo, dinámica trófica, anura, hormigas, ácaros, Caribe.

\section{INTRODUCCIÓN}

El tiempo, espacio y el alimento son algunas de las dimensiones más abordadas en el estudio del nicho de las
Posso-Peláez, C., Blanco-Torres, A., \& Gutiérrez-Moreno, L.C. (2017). Microhabitat use, daily activity and diet of Dendrobates truncatus (Cope, 1861) (Anura: Dendrobatidae) in tropical dry forest in northern Colombia. Acta Zoológica Mexicana (n. s.), 33(3), 490-502.

ABSTRACT. The use of microhabitats, daily activity and diet of three populations of Dendrobates truncatus in the tropical dry lowland forest of the Colombian Caribbean, using the method of visual and manual capture meeting was studied. The study was conducted between March and November, during the three climatic periods: dry, minor and higher rains. Seven microhabitats used were identified and sampled, the activity was measured using the relative number of individuals/hour in four hours daily, stomach contents of anurans were examined and percentage analysis method was applied. D. truncatus has increased activity in the morning (8:00 to 10:00) and late afternoon (16:00 to 18:00) and uses seven types of microhabitats, preferably forest cover and higher humidity. This species presents preference for the consumption of ants especially the family Myrmicinae and the genus Pheidole. These results suggest that the conservation of tropical dry forest relicts and developing rehabilitation strategies for it constitute fundamental elements for the survival of this species.

Key words: Space, time, trophic dynamics, anura, formicidae, ants, mites, Caribe.

especies, y pueden ser estudiadas como recursos (Kronfeld-Schor \& Dayan, 2003; Dias \& Rocha, 2007; Navarro et al., 2013), la forma en que las especies los explotan y dividen permite comprender sus patrones de distribución, 
abundancia y diversidad específica (Toft, 1985). Los estudios sobre el estado y uso de los hábitats por anfibios además de brindar elementos importantes para el conocimiento de la historia natural y ecología de estas especies, han aportado elementos para entender el grado de amenaza actual del grupo; además, se ha determinado cual es la principal causa de pérdida de especies (Isaacs \& UrbinaCardona, 2011; Deichmann et al., 2012; Cortés-Gómez et al., 2013). El tiempo es otra dimensión importante en la partición del nicho, las diferencias en los patrones diarios de actividad constituye un valor adaptativo y la utilización diaria temporal permite la coexistencia exitosa de especies, ya sea entre competidores como entre presas y depredadores (Kronfeld-Schor \& Dayan, 2003).

La familia Dendrobatidae es conocida por poseer productos tegumentarios tóxicos como defensa en contra de predadores en la mayoría de sus especies (Myers et al., 1991). Su alta toxicidad a menudo está asociada con coloración aposemática (coloración vistosa de advertencia) y con una dieta especializada en hormigas, al parecer los alcaloides los obtienen de hormigas y otros insectos consumidos como parte de su dieta (Lötters et al., 2007). Son generalmente de hábitos diurnos y se caracterizan por ser predadoras muy activas (Lötters et al., 2007).

Muchos estudios en anfibios indican que la disponibilidad de presas y la dieta de los anfibios, llegan a exhibir cambios espacio-temporales en diversos ecosistemas (Toft, 1981; Mac Nally, 1983; Donelly, 1991). Debido a sus hábitos terrestres de muchas de sus especies, principalmente en la hojarasca, los dendrobátidos, tienen acceso a una gran variedad de artrópodos e insectos que habitan en el sotobosque (Stork \& Blackburn, 1993).

Dendrobates truncatus tiene una distribución restringida en Colombia, se distribuye en el flanco este de la cordillera occidental y el flanco oeste de la cordillera central en tierras bajas del Caribe, en el rango altitudinal se da desde los 70 hasta los 1200 msnm (Gualdrón-Duarte et al., 2016). Es una especie terrestre y diurna que tolera condiciones extremas de temperatura y humedad. Es posible encontrarla en hojarasca, debajo de piedras o troncos caídos cerca a cuerpos de agua. Los huevos son terrestres y los renacuajos son cargados por los adultos quienes los depositan en charcos temporales, bromelias, huecos con agua de árboles entre otros, para su desarrollo, la reproducción ocurre a lo largo del año con un pico en la estación seca (Lötters et al., 2007; Gualdrón-Duarte et al., 2016). Esta especie es muy utilizada como mascota debido a la relativa facilidad de su cuidado en cautiverio, por tal motivo se encuentra dentro del apéndice II de la Convención sobre el Comercio Internacional de Especies Amenazadas de Fauna y Flora Silvestres (CITES) y presenta categoría de bajo riesgo en la lista roja de IUCN (Castro \& Lynch, 2004; Gualdrón- Duarte et al., 2016).

Existen muchos vacíos de conocimiento sobre autoecología y funciones ecológicas de muchas de las especies de anfibios, si bien existen estudios que mencionen los hábitos alimenticios de ciertas especies de dendrobátidos (e.g. Silverstone, 1975; Donelly, 1991; Toft, 1995; Menéndez-Guerrero, 2001), hasta el momento no se han reportado detalles sobre la composición de la dieta y hábitos alimentarios de $D$. truncatus en su ambiente natural. De igual forma, los estudios correspondientes a los microhábitats utilizados por esta especie en el Caribe colombiano se limitan al trabajo realizado por Cuentas (1999). Por tal motivo, esta investigación describe el uso del espacio a nivel de microhábitats, la variación en la actividad diurna y la dinámica espacial (sitios) y temporal (épocas climáticas) en la dieta de D. truncatus. Se aborda el estudio en una escala regional para áreas con bosque seco tropical de tierras bajas $(<500 \mathrm{msnm})$ del norte de Colombia, bioma que ha perdido la mayor parte de su cobertura original, donde solo quedan fragmentos aislados inmersos en una matriz antropizada (Pizano \& García, 2014).

\section{MATERIALES Y MÉTODOS}

Área de Estudio. El estudio se realizó en tres sitios de muestreo del norte de Colombia, dentro del ecosistema de bosque seco tropical. Sitio 1: Finca Kalashe Kalabia, departamento del Magdalena $\left(11^{\circ} 16.5^{\prime} \mathrm{N}, 74^{\circ} 0.5 \mathrm{~W}\right)$, en las estribaciones de la Sierra Nevada de Santa Marta, 144 $\mathrm{m}$ de elevación, temperatura promedio: $27.29^{\circ} \mathrm{C}$, máxima: $30{ }^{\circ} \mathrm{C}$ - mínima: $25.4{ }^{\circ} \mathrm{C}$. La humedad relativa promedio de 83.17\%, máxima: 99\%; Sitio 2: Los Mameyales, municipio de Piojó- Atlántico ( $10^{\circ} 45^{\prime} 24,80^{\prime}$ ' N, $75^{\circ} 06^{\prime}$ $45.83^{\prime}$ ' W), $253 \mathrm{~m}$ de elevación, temperatura promedio: $26.72{ }^{\circ} \mathrm{C}$, máxima: $34.5^{\circ} \mathrm{C}$ - mínima: $23.5^{\circ} \mathrm{C}$. Humedad relativa promedio: $87.05 \%$, máxima: $99 \%$; Sitio 3: Reserva Campo Alegre, municipio Los Córdobas-Córdoba ( $\left.8^{\circ} 47.9^{\prime} \mathrm{N}, 76^{\circ} 19.6^{\prime} \mathrm{W}\right), 137 \mathrm{~m}$ de elevación, temperatura promedio: $26.28{ }^{\circ} \mathrm{C}$, máxima: $38.1{ }^{\circ} \mathrm{C}$ - mínima: 24.2 ${ }^{\circ} \mathrm{C}$. La humedad relativa promedio: $87.63 \%$, máxima: 99\% (Fig. 1).

Diseño de muestreo. Se llevaron a cabo tres muestreos por sitio (9 eventos en total); estos se ejecutaron entre marzo a noviembre de 2007, debido a que el bosque seco tropical presenta una marcada temporalidad (Pizano \& 


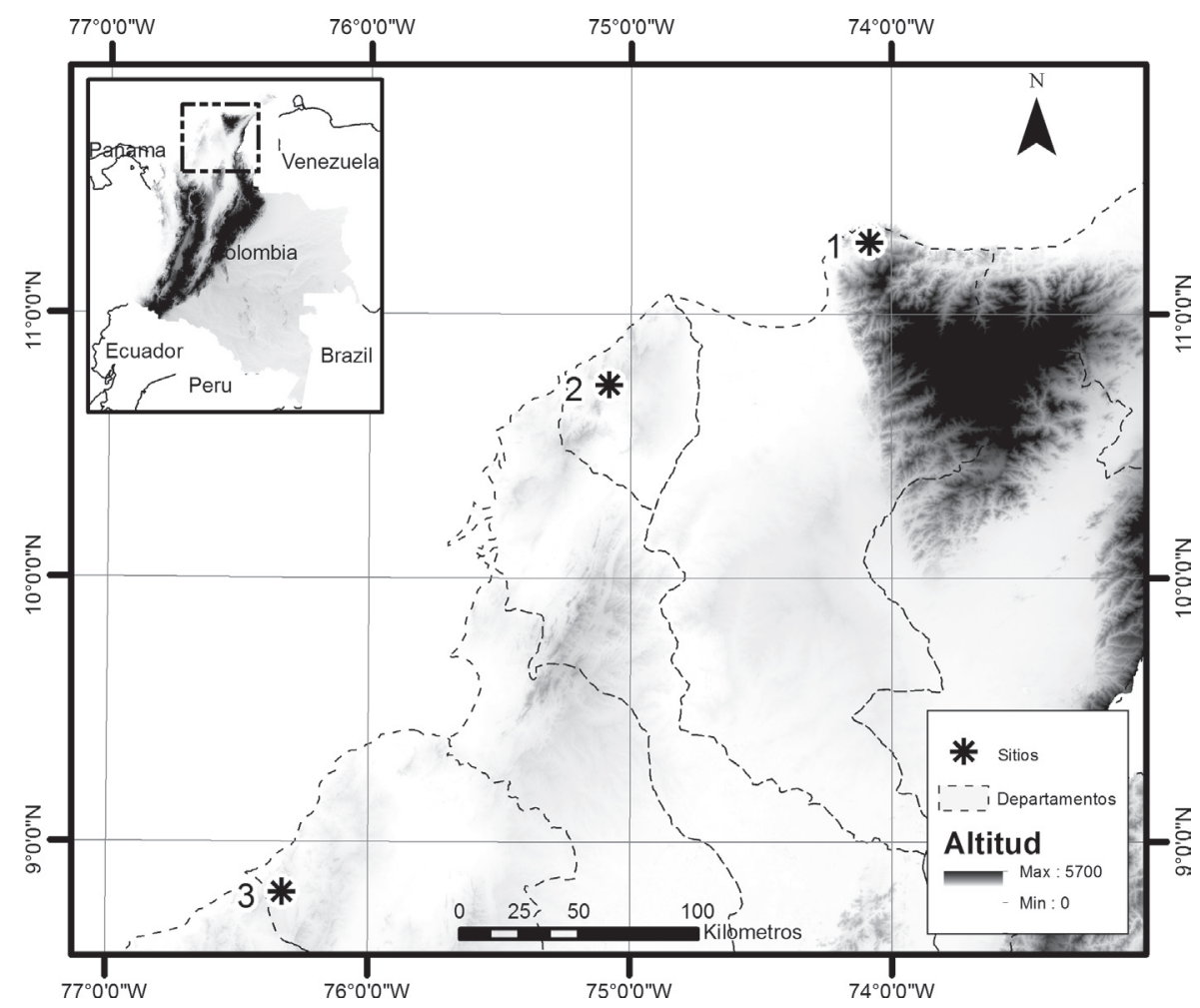

Figura 1. Ubicación de los sitios de estudio de Dendrobates truncatus en bosque seco tropical del norte de Colombia. $1=$ Sitio 1 Finca Kalashe Kalabia, departamento del Magdalena, 2= Sitio 2 Los Mameyales, municipio de Piojó- Atlántico, 3= Sitio 3 Reserva Natural de la Sociedad

Civil Campo Alegre, municipio Los Córdobas - Córdoba.

García, 2014) fue posible muestrear tres épocas climáticas diferentes que correspondieron a la menor precipitación "seco" (marzo), primeras lluvias "lluvias menores" (mayo-junio) y temporada de precipitaciones más abundantes "lluvias mayores" (octubre-noviembre). Para la colecta de datos se utilizó el método de búsqueda por conteos visuales (Heyer et al., 2001; Blanco-Torres \& Bonilla, 2010).

La captura de ejemplares se desarrolló mediante búsqueda activa y captura manual. En el transcurso del día se muestreó en cuatro ocasiones: mañana (8:00 a 10:00), medio día (12:00 a 14:00), tarde (16:00 a 18:00) y noche (19:00 -21:00). En cada caso con una duración de 5 días, por tanto el esfuerzo muestreal fue de 360 horas. Las tasas de encuentro se definieron como el número de individuos de la especie respecto al tiempo (horas) invertido en cada visita (Angulo et al., 2006) y fueron calculadas para cada microhábitat en los horarios diarios establecidos dentro del muestreo.

En cada sitio se seleccionaron distintos microhábitats siguiendo los criterios sugeridos por Ferrer \& González (2002) y Blanco-Torres \& Bonilla (2010): ausencia o presencia de cuerpos de agua (temporales o permanentes), vegetación acuática o terrestre, uso del suelo, cobertura vegetal e inclinación de terreno $>15 \%$ pendiente fuerte, $<15 \%$ pendiente suave. Esto permitió establecer siete microhábitats utilizados por D. truncatus: Interior de Bosque (INT. BQ) que presenta estratificación en tres niveles, en donde el dosel está representado con árboles que alcanzan alturas entre 10 y $25 \mathrm{~m}$, todos los fragmentos superan las 90 ha en tamaño; Caminos de Bosque (CAM. $\mathrm{BQ})$ son senderos construidos que penetran al interior del bosque para desarrollar actividades de investigación, extracción de madera, caza o simplemente senderos para transitar; Ribera de Arroyo Permanente con Pendiente Fuerte (RAPCP) riberas de pequeños arroyos que en época seca mantienen agua corriente, de suelos tipo arcilloso que mantienen una alta humedad, presentan pendientes mayores a 15\% y ejemplares de árboles de gran porte y arbustos fuertemente enraizados; Ribera Arroyo Permanente de Pendiente Suave (RAPSP) a diferencia de las RAPCP los suelos son areno-arcillosos y presentan pendientes menores a $15 \%$; Ribera Arroyo Intervenido Permanente de Pendiente Suave (RAIPSP) son riberas de arroyos permanentes fuertemente intervenidos por la acti- 
vidad propia de los cultivos y el pastoreo, cuyo bosque de galería presenta alta intervención; Zona de Cultivo (ZC) utilizadas principalmente para el cultivo transitorio, en las cuales se pueden formar charcos efímeros utilizados como resguardo temporal de los anuros, presentan modificación continua por quema y preparación de tierras, generalmente no ocupan más de 2 hectáreas de terreno; Zona de Uso Intensivo (ZUI) utilizadas principalmente para vivienda, establos y todas las áreas de labores de la población local, altamente perturbadas por construcciones, la vegetación predominante son la plantas herbáceas que invaden las zonas despejadas.

Análisis de contenido estomacal. Cada individuo capturado fue debidamente identificado con un número de campo (Angulo et al. 2006), registrándosela hora de captura y actividad (canto, reposo, amplexus, entre otros). Los mismos fueron sacrificados in situ, utilizando solución de etanol al 10\% aproximadamente (con el objeto de conservar contenido estomacal hasta llevar a laboratorio), para su fijación se utilizó formol al 10\% y posteriormente para su preservación etanol al 75\%. El contenido estomacal de cada espécimen fue separado por tipo de presa y clasificado hasta alcanzar la menor categoría taxonómica posible, en la mayoría de los casos se pudo identificar a género con ayuda de especialistas (Instituto de Ciencias naturales- Universidad Nacional de Colombia; Departamento de Biología-Universidad del Atlántico) y empleando bibliografía especializada. Se identificaron familias, géneros y morfoespecies o morfotipos además del material acompañante o indeterminado. Para aquellos insectos que presentaron metamorfosis huevo-larva-adulto, cada estadio de desarrollo fue considerado como ítem alimentario diferente debido a la marcada diferencia de hábitos y formas que presentan.

Análisis de los datos. Se determinó la abundancia relativa de la especie en cada microhábitat por temporada del año, esta es la relación de la abundancia observada en los recorridos por microhábitat respecto a la abundancia total observada por temporada. Para el análisis de uso espacial se determinaron porcentajes de número de individuos observados en cada microhábitat por época y sitio de muestreo.

Con un análisis de la estructura de la dieta se calculó la cobertura de cada tipo de presa mediante el conteo de área ocupada, este consiste en colocar cada tipo de presa sobre una cuadrícula de papel milimetrado y contar el número de cuadros que ocupa, de esta manera se obtiene una medida de área expresada en $\mathrm{mm}^{2}$ que puede ser catalogada como cobertura (Tresierra \& Culquichicon, 1993). Luego se estandarizaron los datos a porcentaje, para comparar los aportes de los diferentes organismos que constituyen el contenido alimentario, según la presencia y ocurrencia lo que permite estimar el porcentaje de cada uno de ellos, estos métodos fueron aplicados individualmente en cada contenido estomacal (Tresierra \& Culquichicon, 1993; Blanco-Torres et al., 2014). La frecuencia de ocurrencia se calculó como el número de veces que aparece un tipo de presa en todos los contenidos revisados.

Para explorar diferencias entre las observaciones por microhábitats, tasas de encuentro y estructura de la dieta se aplicó el método no paramétrico de Kruskall-Wallis ya que los datos no cumplieron los supuestos de normalidad (Kolmogorov-Smirnov), esto fue ejecutado en el software Past (Hammer et al., 2001).

\section{RESULTADOS}

Abundancia y microhábitats. Se registraron 277 individuos, el Sitio 1 fue el de mayor abundancia con el $58.12 \%$ (161 individuos) del total, seguido por el Sitio 3 con el $31.41 \%$ ( 87 individuos) y por último el Sitio 2 con el $10.47 \%$ (29 individuos). En el Sitio $1 D$. truncatus se registró en la mayor cantidad de microhábitats (57.1\%), seguido por el Sitio $2(42.8 \%)$ y el Sitio 3 presentó con el $28.5 \%$ (Cuadro 1).

No hubo diferencias en la abundancia durante las diferentes épocas climáticas $(H=2.38, p=0.3)$. El mayor registro de abundancia fue en la época seca con $57 \%$ de los registros, seguido por lluvias mayores con $29.2 \%$ y por último lluvias menores con $13.8 \%$. Esta variación fue estadísticamente significativa entre los diferentes microhábitats $(H=14.19, p=0.02)$, RAPPS fue el microhábitat con mayor abundancia (43.7\%) seguido por el INT. BQ. Los microhábitats ZC, ZUI y RAPCP presentaron la menor abundancia (Cuadro 1).

Tasas de encuentro y microhábitats. Las mayores tasas de encuentro se observaron en las horas de la mañana y tarde; D. truncatus no presentó actividad nocturna (Cuadro 1 y Fig 2). La mayor tasa de encuentro se presentó en RAPSP, seguida por INT. BQ, la menor tasa de encuentro se presentó en ZC y ZUI. En época seca el microhábitat con la mayor tasa de encuentro fue el RAPSP seguido por INT. BQ, en RAPCP no se obtuvo registro de individuos. Solo en esta época fue donde se registraron individuos en los microhábitats ZC y ZUI. Para lluvias menores la mayor tasa fue la de INT. BQ seguida por RAPSP y RAIPSP, los demás microhábitats no tuvieron registros 
Cuadro 1. Variación de la abundancia y tasas de encuentro para Dendrobates truncatus en bosque seco tropical del norte de Colombia.

INT. BQ: Interior de Bosque, CAM. BQ: Caminos de Bosque, RAPCP: Ribera de Arroyo Permanente con Pendiente Fuerte,

RAPSP: Ribera Arroyo Permanente de Pendiente Suave, RAIPSP: Ribera Arroyo Intervenido Permanente de Pendiente Suave, ZC: Zona de Cultivo, ZUI: Zona de Uso Intensivo.

\begin{tabular}{|c|c|c|c|c|c|c|c|c|}
\hline \multirow[t]{2}{*}{ Microhábitat } & \multirow[t]{2}{*}{ Abundancia } & \multicolumn{3}{|c|}{ Abundancia época climática } & \multicolumn{4}{|c|}{ Tasa de encuentro } \\
\hline & & Seco & $\begin{array}{c}\text { Lluvias } \\
\text { menores }\end{array}$ & $\begin{array}{c}\text { Lluvias } \\
\text { mayores }\end{array}$ & Mañana & Mediodía & Tarde & Noche \\
\hline CAM BQ & 7 & 3.16 & & 2.47 & 1.67 & & 0.67 & 0 \\
\hline RAPCP & 1 & & & 1.23 & 0.33 & & & 0 \\
\hline $\mathrm{ZC}$ & 2 & 1.27 & & & 0.67 & & & 0 \\
\hline ZUI & 2 & 1.27 & & & & & 0.67 & 0 \\
\hline
\end{tabular}

para este período. Durante las lluvias mayores el mayor registro fue en el INT. BQ seguido por RAPSP, los microhábitats RAIPSP, ZC y ZUI no tuvieron registro para este período (Cuadro 1).

Dieta. Se examinaron 66 individuos, provenientes de los tres sitios de muestreos, de los cuales el $98.5 \%$ se encontraron con estómago lleno. Se identificaron 45 familias (incluidas morfofamilias) y 121 morfotipos de presas, además de material indeterminado. La clase Insecta fue la de mayor contribución a la dieta identificándose 11 órdenes (84 morfotipos), seguido por Arachnida con dos órdenes (31 morfotipos), Diplopoda (1 morfotipo), Malacostraca (1 morfotipo), Gastropoda (1 morfotipo). Con respecto al porcentaje de cobertura en la dieta los insectos aportaron el $80 \%$, seguidos por arácnidos con $9.5 \%$. Dentro de la clase Insecta el orden con la mayor cantidad de biomasa fue Hymenoptera con el $67.7 \%$, especialmente la familia Formicidae con el $66.8 \%$ del aporte en cobertura a la dieta de D. truncatus. El orden Acari representa la totalidad del aporte de la clase Arácnida y el $9.5 \%$ en la dieta de la especie. Por último, el orden Coleóptera aporta con el 5.5\% de cobertura de la dieta (Cuadro 2).

La estructura de la dieta de Dendrobates truncatus no presentó variaciones marcadas a nivel de orden entre sitios y época climática, en todos los casos Formicidae aportó la mayor parte para su dieta (Cuadro 2). La subfamilia Myr-

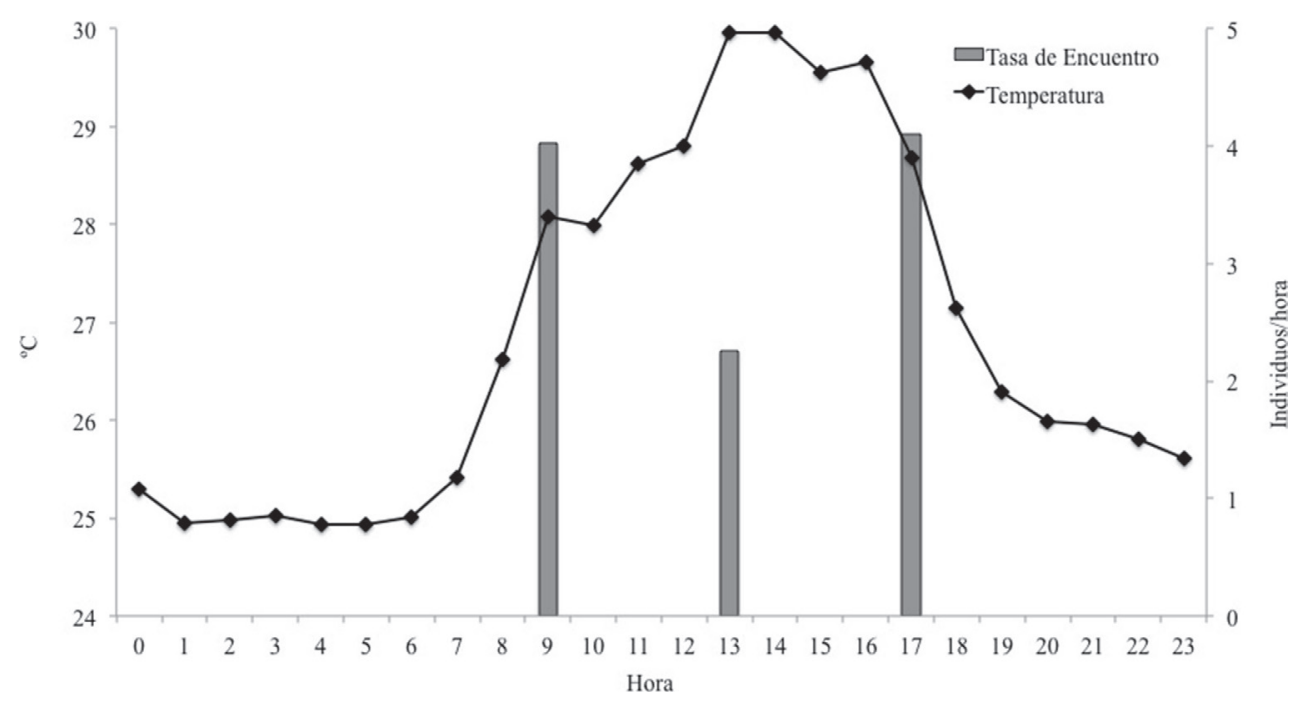

Figura 2. Comportamiento de la temperatura y actividad diaria de Dendrobates truncatus en bosque seco tropical del norte de Colombia. Los valores graficados son el promedio de todas las observaciones de cada variable en horario diario. 
Cuadro 2. Composición de la dieta, cobertura y frecuencia de ocurrencia de presas consumidas por Dendrobates truncatus en bosque seco tropical del norte de Colombia.

\begin{tabular}{|c|c|c|c|c|c|c|c|}
\hline \multirow[t]{2}{*}{ Categoría Presa } & \multirow{2}{*}{$\begin{array}{l}\text { Frecuencia } \\
\text { de ocurrencia }\end{array}$} & \multicolumn{6}{|c|}{ COBERTURA (\%) } \\
\hline & & Seco & Ll. men & Ll. may & Sitio 1 & Sitio 2 & Sitio 3 \\
\hline Arachnida (Acari) & 0,72 & 7,25 & 7,81 & 4,81 & 9,84 & 7,55 & 3,82 \\
\hline Caraboidodea sp1 & 0,02 & 0,18 & & & 0,14 & & \\
\hline Ceratozetoidea sp1 & 0,05 & 0,42 & & & & & 0,32 \\
\hline Eremaeoidea sp1 & 0,14 & 0,60 & 1,03 & & 1,26 & 0,27 & 0,18 \\
\hline Eremaeoidea sp2 & 0,08 & 0,36 & 0,72 & & 0,14 & & 0,86 \\
\hline Eremuloidea sp1 & 0,25 & 2,28 & 0,76 & 4,54 & 1,36 & 7,82 & 0,95 \\
\hline Eremuloidea sp2 & 0,14 & 1,86 & 1,12 & & 2,20 & 0,13 & 0,36 \\
\hline Eremuloidea sp3 & 0,05 & 0,12 & 0,13 & & 0,23 & & \\
\hline Euposidae sp1 & 0,02 & 0,06 & & & & & 0,05 \\
\hline Galumnoidea sp1 & 0,09 & 0,06 & 1,07 & & 0,47 & 0,66 & 0,45 \\
\hline Gymnodamaeoidea sp1 & 0,03 & & 0,18 & & 0,14 & 0,13 & \\
\hline Hermannielloidea sp1 & 0,02 & & 0,04 & & & & 0,05 \\
\hline Heterochihonoidea sp1 & 0,05 & 0,30 & 0,04 & & & 0,13 & 0,23 \\
\hline Hydrozetoidea sp1 & 0,02 & & 0,09 & & 0,09 & & \\
\hline Lealapidae sp1 & 0,06 & 0,24 & & & 0,05 & & 0,14 \\
\hline Liacaroidea sp1 & 0,03 & 0,12 & & & 0,05 & 0,13 & \\
\hline Oppioidea sp1 & 0,02 & 0,06 & & & 0,05 & & \\
\hline Oribatuloidea sp1 & 0,11 & 0,18 & 1,56 & 0,17 & 1,54 & 0,66 & 0,09 \\
\hline Trombidiidae sp1 & 0,09 & 0,18 & 1,34 & 0,59 & 0,56 & 1,33 & 0,82 \\
\hline Trombidiidae sp2 & 0,02 & & & 0,50 & & & 0,27 \\
\hline Uropodidae sp1 & 0,05 & 0,24 & 0,04 & & 0,23 & & \\
\hline Acari sp1 & 0,18 & 1,92 & 0,04 & 1,01 & 1,59 & & 0,50 \\
\hline Acari sp2 & 0,03 & & & 0,17 & 0,05 & & 0,05 \\
\hline Acari sp3 & 0,05 & 0,06 & & 0,34 & 0,19 & & 0,05 \\
\hline Acari sp4 & 0,02 & & & 0,17 & 0,09 & & \\
\hline Acari sp5 & 0,03 & & 0,13 & 0,25 & 0,28 & & \\
\hline Acari sp6 & 0,03 & 0,36 & & & 0,23 & & 0,05 \\
\hline Acari sp7 & 0,02 & & 0,18 & & 0,19 & & \\
\hline Acari sp8 & 0,03 & 0,06 & 0,76 & & 0,80 & & 0,05 \\
\hline Acari sp9 & 0,02 & 1,38 & & & 1,08 & & \\
\hline Acari sp10 & 0,02 & 0,06 & & & & & 0,05 \\
\hline Arachnida(Scorpiones) & 0,03 & 0,18 & & & & & 0,14 \\
\hline Scorpiones sp & & & & & & & \\
\hline Insecta (Coleoptera) & 0,47 & 6,15 & 2,60 & 3,03 & 5,88 & 0,89 & 3,41 \\
\hline Anobiidae sp1 & 0,02 & 0,30 & & & & & 0,23 \\
\hline Anobiidae sp2 & 0,02 & 0,06 & & & & & 0,05 \\
\hline Bostrichidae sp & 0,02 & & & 0,42 & & & 0,23 \\
\hline Carabidae sp & 0,02 & & 0,27 & & 0,28 & & \\
\hline Coccinaelidae sp & 0,05 & 0,72 & & & 0,23 & & 0,32 \\
\hline Curculionidae sp1 & 0,02 & 0,06 & & & & & 0,05 \\
\hline Curculionidae sp2 & 0,02 & & & 0,34 & & & 0,18 \\
\hline
\end{tabular}


Cuadro 2. Continúa.

\begin{tabular}{|c|c|c|c|c|c|c|c|}
\hline \multirow[t]{2}{*}{ Categoría Presa } & \multirow{2}{*}{$\begin{array}{c}\text { Frecuencia } \\
\text { de ocurrencia }\end{array}$} & \multicolumn{6}{|c|}{ COBERTURA (\%) } \\
\hline & & Seco & Ll. men & L1. may & Sitio 1 & Sitio 2 & Sitio 3 \\
\hline Arachnida (Acari) & 0,72 & 7,25 & 7,81 & 4,81 & 9,84 & 7,55 & 3,82 \\
\hline Histeridae sp & 0,02 & 0,00 & 0,40 & & & & 0,41 \\
\hline Nitidulidae sp1 & 0,02 & 0,24 & & & 0,19 & & \\
\hline Pselaphidae sp1 & 0,06 & 1,62 & & & 1,17 & & 0,09 \\
\hline Psephenidae sp & 0,02 & & 0,18 & & 0,19 & & \\
\hline Psephenidae (Larva) & 0,02 & 1,56 & & & & & 1,18 \\
\hline Scydmaenidae sp & 0,11 & 2,58 & 0,18 & & 1,96 & 0,13 & 0,18 \\
\hline Staphylinidae sp4 & 0,02 & 0,18 & & & 0,14 & & \\
\hline Staphylinidae sp5 & 0,02 & & & 0,25 & 0,14 & & \\
\hline Staphylinidae sp6 & 0,03 & & 0,85 & & 0,89 & & \\
\hline Staphylinidae sp7 & 0,03 & & 0,54 & 1,09 & 1,17 & & \\
\hline Staphylinidae sp8 & 0,02 & & 0,09 & & 0,09 & & \\
\hline Staphylinidae sp9 & 0,02 & & & 0,50 & & & 0,27 \\
\hline Staphylinidae sp10 & 0,02 & & & 0,84 & & & 0,45 \\
\hline Staphyinidae sp11 & 0,02 & & & 0,25 & 0,14 & & \\
\hline Isotomidae sp & 0,03 & 0,78 & & & 0,51 & & 0,09 \\
\hline Insecta (Diptera) & 0,13 & 2,00 & 0,53 & 0,57 & 2,16 & & 0,47 \\
\hline Cecidomyidae (Larva) & 0,02 & 0,12 & & & 0,09 & & \\
\hline Forcipomyia sp & 0,02 & 2,46 & & & 1,92 & & \\
\hline Ceratopogonidae sp & 0,03 & & 0,63 & & 0,65 & & \\
\hline Ceratopogonidae Larva & 0,02 & 0,06 & & & & & 0,05 \\
\hline Diptera sp1 & 0,06 & 0,12 & & 0,92 & & & 0,59 \\
\hline Diptera sp2 & 0,02 & 0,24 & & & 0,19 & & \\
\hline Psychodidae sp & 0,02 & 0,06 & & & & & 0,05 \\
\hline Insecta (Hemiptera) & 0,05 & 1,62 & & & 0,05 & 3,45 & \\
\hline \multicolumn{8}{|l|}{ Lygaeidae sp } \\
\hline Insecta (Hymenoptera- Formicidae) & 0,95 & 54,96 & 74,68 & 62,75 & 58,46 & 53,73 & 73,49 \\
\hline Acantognathus sp & 0,02 & 0,54 & & & & & 0,41 \\
\hline Apterostigma sp & 0,05 & 0,12 & 0,13 & & 0,09 & & 0,14 \\
\hline
\end{tabular}


Cuadro 2. Continúa.

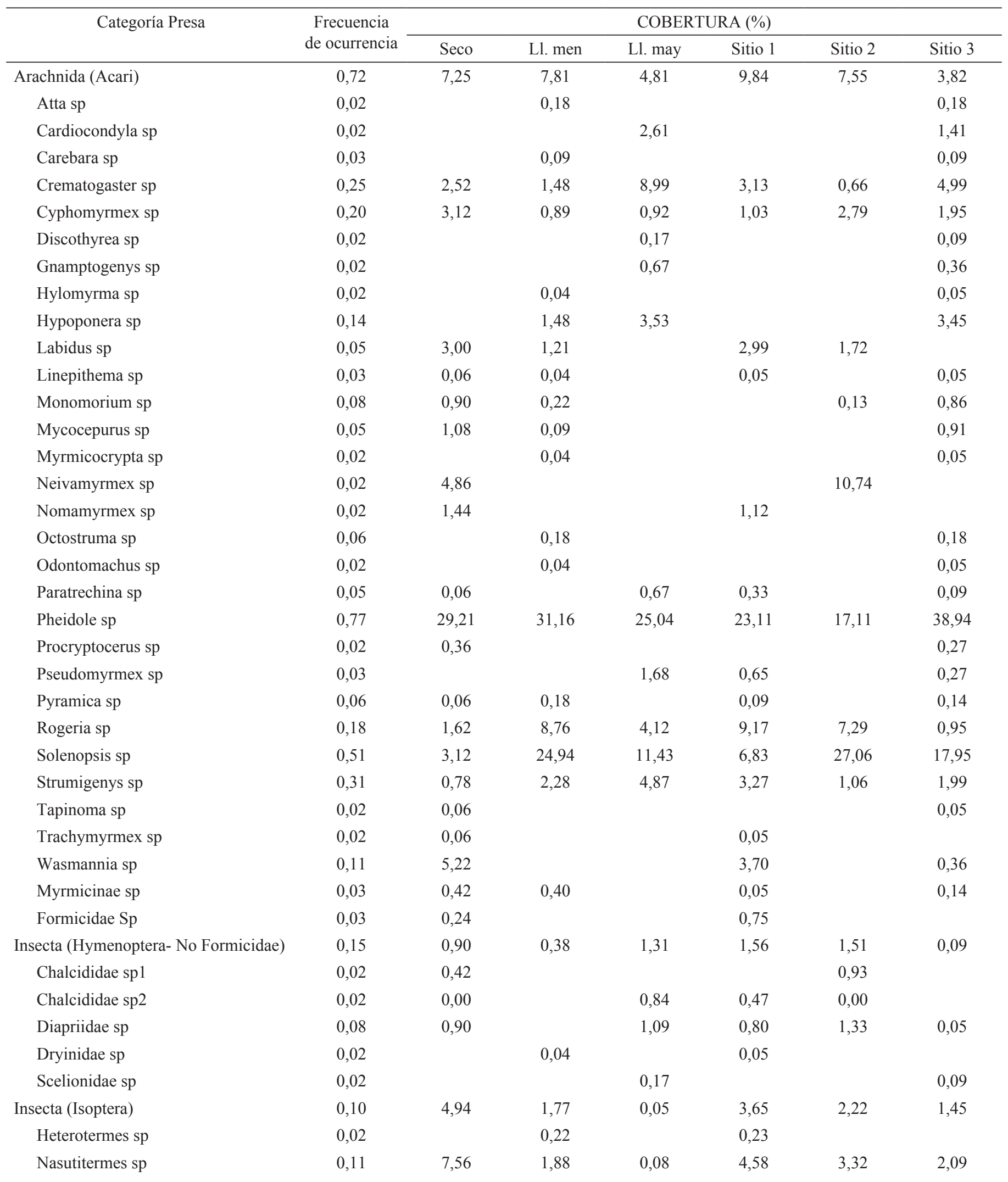


Cuadro 2. Continúa.

\begin{tabular}{|c|c|c|c|c|c|c|c|}
\hline \multirow[t]{2}{*}{ Categoría Presa } & \multirow{2}{*}{$\begin{array}{c}\text { Frecuencia } \\
\text { de ocurrencia }\end{array}$} & \multicolumn{6}{|c|}{ COBERTURA (\%) } \\
\hline & & Seco & Ll. men & Ll. may & Sitio 1 & Sitio 2 & Sitio 3 \\
\hline Arachnida (Acari) & 0,72 & 7,25 & 7,81 & 4,81 & 9,84 & 7,55 & 3,82 \\
\hline Psocoptera sp & 0,03 & 0,12 & & & & & 0,09 \\
\hline Insecta (Thysanoptera) & 0,06 & 0,30 & & & 0,05 & & 0,18 \\
\hline Larva & 0,24 & 0,71 & 7,17 & 7,63 & 7,29 & 0,18 & 4,61 \\
\hline Larva sp1 & 0,02 & 0,12 & & & 0,09 & & \\
\hline Larva sp2 & 0,02 & 0,42 & & & 0,33 & & \\
\hline Larva sp3 & 0,22 & 0,54 & 8,49 & 12,27 & 9,21 & 0,27 & 6,62 \\
\hline Diplopoda & 0,12 & 0,78 & 1,17 & 3,24 & 1,20 & 4,17 & 1,01 \\
\hline \multicolumn{8}{|l|}{ Oniscidea sp } \\
\hline Indeterminado & 0,11 & 0,66 & 0,54 & 0,25 & 0,75 & 0,93 & 0,14 \\
\hline
\end{tabular}

micinae fue la categoría de presa con mayor importancia a nivel de cobertura (91\%) y riqueza $(63.6 \%)$ de la familia Formicidae, seguida por Ponerinae con $2.5 \%$ de aporte en cobertura. Las otras subfamilias (Pseudomyrmicinae, Formicinae y Dolichoderinae) representaron menos del $1 \%$ de la cobertura. A nivel de género, Pheidole aportó el $43.5 \%$ de la cobertura aportada por la familia y el $60.8 \%$ del porcentaje total de la dieta, seguido por los individuos del género Solenopsis $(21.9 \%)$ y Rogeria $(9.9 \%)$ respecto a la cobertura aportada por Formicidae.

Se presentaron diferencias significativas entre sitios a nivel de morfotipo de presa consumida $(\mathrm{H}=31.77$, $p<0.05)$. Los Sitios 1 y 3 presentaron la mayor riqueza de presas consumidas con 78 y 79 morfotipos respectivamente, seguidos por el Sitio 2 con una riqueza de 30 morfotipos. El género Pheidole fue el ítem alimenticio de mayor contribución a la dieta de D. truncatus presentando porcentajes de cobertura mayores al $18 \%$ en los tres sitios y siendo aún más representativa en el Sitio 3 llegando al $34.14 \%$ de la cobertura.

Hubo diferencias significativas en la dieta de D. truncatus entre las épocas climáticas $(H=17.02, p<0.05)$ a nivel de morfotipo de presa consumida. La época seca aportó la mayor cantidad de ítems a la dieta de $D$. truncatus con 81 ítems, seguida por lluvias menores (55 items) y lluvias mayores con 42 items. El ítem con mayor apor- te fue Pheidole con porcentajes de cobertura mayores al $25 \%$ y fue consumido durante las tres épocas climáticas.

\section{DISCUSIÓN}

La mayor complejidad en la cobertura vegetal (la cual disminuye la temperatura y exposición a la radiación solar dentro del hábitat) y la presencia de agua son factores determinantes en la dispersión y actividad de los anuros (Woolbright, 1985; Navas, 1996; Dodd, 2010), por otro lado la producción de hojarasca en ambientes boscosos y húmedos dentro del bosque aumenta la oferta de alimento respecto a las áreas intervenidas (Vásquez-Vélez et al., 2013). En bosque seco tropical, estos factores generan ambientes óptimos para el establecimiento de $D$. truncatus, es por esto que los microhábitats de interior de bosque y ribera de cuerpos de agua permanentes registran una mayor abundancia contrastando con microhábitats muy intervenidos. La abundancia de esta especie no está determinada por la cantidad de área ofertada, sino por la calidad de la misma, entendiendo como calidad las condiciones que oferte el microhábitat para lograr un sostenimiento de los requerimientos de la especie (humedad, calidad de agua, heterogeneidad vertical, oferta alimenticia, entre otros (Cortés-Gómez et al., 2013). La eliminación de la cober- 
tura vegetal natural e incluso su transformación a áreas de cultivo influyen de manera directa en la disminución de la abundancia relativa de algunas las especies (Cortés-Gómez et al., 2013; Méndez-Narváez \& Bolívar-G, 2016), efecto que se observa al comparar las abundancias por microhábitats de $D$. truncatus en este estudio.

La disminución de las tasas de encuentro en lluvias menores puede estar relacionado a la dispersión de los individuos, adicionalmente los cambios fenológicos del bosque y en especial el aumento de la vegetación herbácea disminuyen la capacidad de avistamiento (Heyer et al., 2001; Angulo et al., 2006). Esta dinámica había sido descrita por Cuentas (1999) para una población de la misma especie en una zona con bosque seco tropical del Caribe colombiano (Santuario de Flora y Fauna los Colorados, Bolívar, Colombia).

La variación climática anual del bosque seco tropical produce migraciones locales de individuos (García et al., 2014) en este caso de D. truncatus. El déficit hídrico y la exposición a la radiación solar, mediada por el fenómeno de defoliación del bosque seco tropical, serían los factores determinantes de la distribución local de las poblaciones de esta especie, produciendo fenómenos de agregación durante la época seca en microhábitats donde la cobertura vegetal y la presencia de agua generan un confort climático para el establecimiento de las poblaciones, lo cual es consistente con los registros de abundancia que se presentan durante esta época en arroyos permanentes e interior de bosques. Durante los períodos de lluvias, cuando se aumenta el área óptima debido al incremento de la humedad o disposición de agua y disminución de la exposición a la radiación por el aumento de la cobertura foliar, se presenta una dispersión de los individuos, esto concuerda con lo propuesto por Cuentas (1999) sobre que los gradientes de humedad en el sustrato son un factor determinante en la expansión (periodo de lluvias) y contracción (periodo de no lluvias) de la distribución de $D$. truncatus a lo largo del año en torno a los arroyos.

En el bosque seco tropical D. truncatus es una especie diurna, concordante con lo descrito para la familia (Graves, 1999; Lötters et al., 2007). Presenta dos picos de actividad, uno matinal y otro vespertino, generándose una disminución de su actividad durante el período de mayor incidencia de radiación solar diaria, que corresponde al comprendido entre las 10:00 a 14:00, esto es una adaptación comportamental para establecerse en el bosque seco tropical y así evitar altas temperaturas (Graves, 1999). Esta condición le permite coexistir con las otras especies de anuros las cuales, en su mayoría, son de hábi- tos nocturnos. Debido a esto, la repartición del tiempo en D. truncatus permite la segregación de su nicho, evita su extinción y sobrevivencia como especialista (Kronfeld \& Dayan, 2003).

Dendrobates truncatus es un forrajero activo especialista en hormigas (especialmente la subfamilia Myrmicinae) y ácaros, lo que concuerda con lo sugerido para otras especies del género y la familia Dendrobatidae (Caldwell, 1996; Lötters et al., 2007; Cajade et al., 2010; Arce \& Rengifo, 2013, Gómez-Hoyos et al., 2014). La alta contribución de hormigas en su dieta es atribuible principalmente a la bioacumulación y transformación de algunos metabolitos secundarios, las cuales han sido estudiadas durante años como posibles precursoras de los venenos producidos por las diferentes especies de la familia Dendrobatidae (Daly et al., 1987; Saporito et al., 2004), lo que ha permitido establecer la conexión entre las Decahydroquinolinas y las Pirrolizidinas, alcaloides comunes entre los myrmicinos y las ranas del género Dendrobates. Las hormigas son un recurso fácilmente disponible para predadores, ya que se encuentran en colonias y con escasas formas aladas, pero poseen un bajo contenido nutricional (Parmelee, 1999), ya que son presas quitinosas de difícil digestión (Toft, 1985) por lo que deben ser consumidas en grandes cantidades.

Las hormigas mirmicinas presentan una diversidad de hábitos muy grande, acorde con la riqueza de especies en la subfamilia. Hay formas arborícolas (como Procryptocerus, Crematogaster) que habitan el suelo y la hojarasca (Strumigenys, Pyramica, Pheidole, Solenopsis), algunas presentan asociaciones con plantas, hongos o con otras hormigas (Crematogaster) (Fernández, 2003). El género Pheidole es uno de los géneros más comunes de la Región Neotropical, habitan principalmente el estrato epígeo y resultan muy abundantes en el área de estudio (Benson \& Brandão, 1987; Fowler et al., 1993).

Algunas especies de los géneros Wasmannia y Solenopsis, son conocidas con el nombre de "pequeñas hormigas del fuego" y constituyen una plaga importante en algunas zonas (Ulloa-Chacón \& Cherix, 1988). Al igual que las Myrmicinae las hormigas representantes de las demás subfamilias presentes en la dieta, pueden ocupar diferentes estratos, desde el arbóreo como las especies del género Tapinoma hasta el suelo tal es el caso de Paratrechina, Hypoponera, Gnamptogenys, Odontomachus) (Fernández, 2003). Otras hormigas pertenecientes a la subfamilia Pseudomyrmicinae poseen especies habitantes obligadas de plantas myrmecófilas, tales como acacias con espinas u otras especies de leguminosas. Las hormi- 
gas de la subfamilia Ecitoninae son insectos populares en muchos lugares del Neotrópico, donde son famosas sus "incursiones de limpieza" a las casas de habitantes de áreas rurales, particularmente en climas cálidos: "Las masivas columnas de especies conspicuas como las de Labidus hacen que las actividades depredadoras de estas hormigas no pasen desapercibidas para las personas que habitan o frecuentan el campo" (Fernández, 2003). De esta forma se observa que a pesar de que D. truncatus es una especie terrestre, en su dieta posee representantes de los diferentes estratos verticales, dando muestra de la complejidad de las zonas donde habita.

El resto de la cobertura de la dieta de D. truncatus es aportada por otras familias de himenópteros como: Chalcididae, Diapriidae, Dryinidae, Scelionidae, todas ellas poseen larvas parásitas de huevos de insectos de los cuales se alimentan. Los adultos se pueden encontrar en follaje, hongos y áreas con vegetación en descomposición (Wolff, 2006), coincidiendo con el la distribución de la especie.

Los ácaros terrestres de vida libre son animales muy diversos y abundantes en medios ricos de materia orgánica, en los árboles, en el suelo y en ambientes húmedos, siendo coincidentes con los hábitos de la especie en estudio. De igual forma estudios realizados por diversos autores, por ejemplo Saporito et al. (2007), muestra que este grupo constituye una de las principales fuentes de las toxinas producidas por dendrobátidos. Por último, el orden Coleóptera también se encuentra formando parte de la dieta de D. truncatus, este es el grupo más grande y diverso de los insectos, lo que permite que se presente algunas presas disponibles para esta especie.

Tanto el régimen de lluvias, como el de sequía influyen en la composición de la dieta de D. truncatus. En zonas tropicales es evidente la variación temporal de poblaciones, algunas especies fluctúan ampliamente, mientras que otras lo hacen de una forma más restringida (Graves, 1999). Se sabe que las hormigas son muy sensibles al contenido de humedad (Levings, 1983), durante las lluvias en donde las inundaciones se hacen presentes, el agua arrastra muchos organismos del suelo o en su defecto los desplaza a zonas más altas donde esta especie no habita, mientras que en sequía se produce lo contrario, los insectos que se encuentran a nivel de suelo aumentan y son más fáciles de capturar. Todo esto influye en la estructura de la dieta de una especie de hábitos terrestres como D. truncatus.

Esta investigación identifica las condiciones específicas para el establecimiento y desarrollo de las poblacio- nes de D. truncatus en el bosque seco tropical por tanto, proporciona elementos importantes para la conservación de la especie. Consideramos necesario el mantenimiento de las condiciones naturales de los bosques relictuales y el aumento de la conectividad estructural entre los mismos para garantizar la supervivencia de la especie y la funcionalidad del bosque seco tropical.

AGRADECIMIENTOS. Agradecemos al grupo de Investigación en Biodiversidad del Caribe Colombiano de la Facultad de Ciencias Básicas de la Universidad del Atlántico, por el apoyo académico (licencias de colección y logística). Pobladores y administradores de los sitios de estudio por el apoyo logístico. Entomólogos especialistas del Instituto de Ciencias Naturales de la Universidad Nacional de Colombia y del Departamento de Biología de la Universidad del Atlántico, por el apoyo en identificación de presas. María C. Franco por el soporte cartográfico.

\section{LITERATURA CITADA}

Angulo, A., Rueda-Almonacid, J. V., Rodriguez-Mahecha, J. V. \& La Marca, E. (Eds). (2006). Técnicas de inventario y monitoreo para los anfibios de la región tropical andina. Conservación Internacional. Serie manuales de campo No 2. Panamericana Formas e Impresos S. A., Bogotá, Colombia, 299 pp.

Arce Domínguez, F. \& Rengifo Mosquera, J. T. (2013). Dieta de Phyllobates aurotaenia y Oophaga histrionica (Anura: Dendrobatidae) en el Municipio del Alto Baudó, Chocó, Colombia. Acta Zoológica Mexicana (n.s), 29, 255-268.

Blanco-Torres, A., Ordoñez, M. P. \& Franco, M. C. (2014). Colostethus ruthveni (Santa Marta Poison Arrow Frog). DIET. Herpetological review, 45, 476.

Blanco-Torres, A. \& Bonilla, M. (2010). Partición de microhábitats entre especies de Bufonidae y Leiuperidae (Amphibia: Anura) en áreas con bosque seco tropical de la región Caribe-Colombia. Acta Biológica Colombiana, 15, 47-60.

Benson, W. \& Brandao, C. (1987). Pheidole diversity in the humid tropics: a survey from Serra dos Carajas, Pará, Brazil, pp. 593594. In: J. Eder \& H. Rembold (Eds.). Chemistry and biology of social insects. Verlag J. Peperny, Munchen, Germany.

Cajade. R., Schaefer, E. F., Duré, M. \& Kehr, A. I. (2010). Trophic and microhabitat niche overlap in two sympatric Dendrobatids from La Selva, Costa Rica. Cuadernos de Herpetología, 24, 81-92.

Caldwell, J. P. (1996). The evolution of myrmecophagy and its correlates in poison frog (Family Dendrobatidae). Journal of the Zoological Society of London, 240, 75-101.

Castro, F. \& Lynch, J. (2004). Dendrobates truncatus. The IUCN Red List of Threatened Species. Version 2014.3. Available at: www.iucnredlist.org (accessed on February 2016).

Cortés-Gómez, A. M., Castro-Herrera, F. \& Urbina-Cardona, J. N. (2013). Small changes in vegetation structure create great changes in amphibian ensembles in the Colombian Pacific rain forest. Tropical Conservation Science, 6(6), 49-769. 
Cuentas, D. (1999). Influencia de la cobertura vegetal en la distribución espacial de Dendrobates truncatus (Anura: Dendrobatidae). Tesis de Grado (Biólogo), Universidad del Atlántico, Colombia. $110 \mathrm{pp}$.

Daly, J. W., Myers, C. W. \& Whittaker, N. (1987). Further classification of skin alkaloids from Neotropical poison frogs (Dendrobatidae), with a general survey of toxin/ noxious substances in the Amphibia. Toxicon, 25, 1023-1095.

Deichmann, J. L., Toft, C., Deichmann, P. M., Lima, A. P. \& Williamson, G. B. (2012). Neotropical primary productivity affects biomass of the leaf-litter herpetofaunal assemblage. Journal of Tropical Ecology, 28, 427-435.

Dias, E. J. \& Rocha, C. F. (2007). Niche differences between two sympatric whiptail lizards (Cnemidophorus abaetensis and $C$. ocellifer, Teiidae) in the resting a habitat of northeastern Brazil. Brazilian Journal of Biology, 67, 41-6

Dodd, K. (Ed.). (2010). Amphibian ecology and conservation. Oxford University Press. Oxford, Great Britain, 556 pp.

Donelly, M. A. (1991). Feeding patterns of strawberry poison frog, Dendrobates pumilio (Anura: Dendrobatidae). Copeia, 3, 723-730.

Fernández, F. (Ed.). (2003). Introducción a las Hormigas de la región Neotropical. Instituto de Investigación de Recursos Biológicos Alexander von Humboldt. Bogotá, Colombia, 411 pp.

Ferrer, J. \& González, M. (2002). Fauna anura presente en los microhabitats disponibles en un transepto entre el río Magdalena y el mar Caribe (Departamento del Atlántico). Tesis de Grado (Biólogo), Universidad del Atlántico, Barranquilla. 120 pp.

Fowler, H. G., Bueno, O. C., Sadatsune, T. \& Montelli, A. C. (1993). Ants as potential vectors of pathogens in hospitals in the state of Sao Paulo, Brazil. Insect Science and its Application, 14, 367-370.

Gómez-Hoyos, D., López-García, M., Soto-Garzón, C., MéndezRojas, D., Kahn, T. \& Velasco, J. año?? Geographic variation in the diet of the Cauca Poison Frog Andinobates bombetes (Anura: Dendrobatidae) in the Andes of Colombia. Herpetology Notes, 7 , 559-564.

Graves, B. (1999). Diel activity patterns of the sympatric poison dart frogs, Dendrobates auratus and D. pumilio, in Costa Rica. Journal of Herpetology, 33(3), 375-81.

Gualdrón-Duarte, J., Luna-Mora, V.F., Rivera-Correa, M. \& Kahn, T. (2016). Yellow-striped Poison Frog Dendrobates truncatus (Cope, 1861 “1860”). pp. 323-327. In: T.R. Kahn, E. La Marca, S. Lötters, J. Brown, E. Twomey \& A. Amézquita (Eds). Aposematic poison frogs (Dendrobatidae) of the andean countries: Bolivia, Colombia, Ecuador, Perú and Venezuela. Conservation International, Arlington.

Hammer, Ø., Harper, D.A.T. \& Ryan, .P. D. (2001). PAST: Paleontological statistics software package for education and data analysis. Palaeontologia Electronica, 4, 1-9.

Heyer, W. R., Donnelly, M. A., McDiarmid, R. W., Hayek, L. C. \& Foster, M. S. (2001). Medición y monitoreo de la diversidad biológica. Métodos estandarizados para anfibios. Editorial Universitaria de La Patagonia. Argentina.

Isaacs, P. \& Urbina-Cardona, J. N. (2011). Anthropogenic disturbance and edge effects on anuran ensembles inhabiting Cloud Forest fragments in Colombia. Natureza e Conservação: Brazilian Journal of Nature Conservation, 9, 39-46.
Kronfeld-Schor, N. \& Dayan, T. (2003). Partitioning of time as an ecological resource. Annual Review of Ecology, Evolution, and Systematics, 34, 153-181

Levings, S. (1983). Seasonal, annual, and among-site variation in the ground ant community of a deciduous tropical forest: some causes of patchy species distributions. Ecological Monographs, 53, 435455

Lötters, S., Jungfer, K., Henkel, F. \& Schmidt, W. (2007). Poison frogs. Biology, Species and Captive Husbrandy. Chimaira Buchhandelsgesellschaft mbH. Franckfurt, Germany. 668 pp.

Mac Nally, R. C. (1983). On assessing the significance of interspecific competition to guild structure. Ecology, 64, 1646-1652.

Méndez-Narváez, J. \& Bolívar-G, W. (2016). Complementary Ecological Approaches to Understand Decreases in Frog Diversity in Altered Andean Ecosystems. South American Journal of Herpetology, 11, 1-11.

Menéndez-Guerrero, P. A. (2001). Ecología trófica de la comunidad de anuros del Parque Nacional Yasuní en la Amazonía Ecuatoriana. Tesis de Grado, Pontificia Universidad Católica del Ecuador, Ecuador.

Myers, C. W., Paolillo, A. \& Daly, J. W. (1991). Discovery of a defensively malodorous and nocturnal frog in the family Dendrobatidae: phylogenetic significance of a new genus and species from the Venezuelan Andes. American Museum Novitates, 3002, 1-33

Navarro, J., Votier, S. C., Aguzzi, J., Chiesa, J. J. \& Forero, M.G. (2013). Ecological segregation in space, time and trophic niche of sympatric planktivorous petrels. Plos One, 8(4), e62897

Navas, C. A. (1996). The effect of temperature on the vocal activity of tropical anurans: a comparison of high and low-elevation species. Journal of Herpetology, 30, 488-497.

Parmelee, J. R. (1999). Trophic ecology of a tropical anuran assemblage. Scientific Papers Natural History Museum University of Kansas, 11, 1-59.

Pizano, C. \& García, H. (Eds). (2014). El Bosque Seco Tropical en Colombia. Instituto de Investigación de Recursos Biológicos Alexander von Humboldt (IAvH). Bogotá, Colombia, 349 pp.

Saporito, R. A., Donnelly, M. A., Norton, R., Garraffo, H. M., Spande, T. F. \& Daly, J. W. (2007). Oribatid mites as a new and significant source of alkaloids in poison frogs. Proceedings of the National Academy of Sciences, 104, 8885-8890.

Saporito, R. A., Garraffo, H. M., Donnelly, M. A., Edward, A. L., Longino, J. T. \& Daly, J. W. (2004). Formicine ants: an arthropod source for the pumiliotoxin alkaloids of dendrobatid poison frogs. Proceedings of the National Academy of Sciences, 101, 8045-8050.

Silverstone, P. A. (1975). A revision of the poison-arrow frogs of the genus Dendrobates Wagler. Natural History Museum of Los Angeles County. Science Bulletin, 21, 1-55.

Stork, N. E. \& Blackburn, T. M. (1993). Abundance, body size and biomass of arthropods in tropical forest. Oikos, 67, 483-489.

Toft, C. A. (1981). Feeding ecology of Panamanian litter anurans: patterns in diet and foraging mode. Journal of Herpetology, 15, 139-144.

Toft, C. A. (1985).Resource partitioning in amphibians and reptiles. Copeia, 1985, 1-21.

Toft, C. A. (1995). Evolution of diet specialization in poison-dart frogs (Dendrobatidae). Herpetologica, 51, 202-216. 
Tresierra, A. E. \& Culquichicon, Z .G. (1993). Manual de Biología Pesquera. Trujillo, Perú, 432 pp.

Ulloa-Chacón, P. \& Cherix, D. (1988). Quelques aspecs de la biologie de Wasmannia auropunctata (Roger) (Hymenoptera, Formicidae). Actes des Colloques Insectes Sociaux, 4, 177-184.

Vásquez-Vélez, A. I., Garzón, S. \& Asencio-Santofimio, H. (2013). Caracterización florística asociada al hábitat de Dendrobatidae (Amphibia: Anura), en la localidad de Piangüi- ta (Bahía de Buenaventura, Pacífico Colombiano). Boletín Científico Centro de Museos Museo de Historia Natural, 17, 17-32.

Woolbright, L. L. (1985). Patterns of nocturnal movement and calling by the tropical frog Eleutherodactylus coqui. Herpetologica, 41, $1-9$.

Wolf, M. (2006). Insectos de Colombia. Laboratorio de Colecciones Entomológicas GIEM. Medellín, Colombia. 